\title{
A alimentação com fórmula em recém-nascidos prematuros ou com baixo peso ao nascer
}

\author{
Formula feeding in preterm \\ or low birth weight newborns
}

\section{Alimentación con fórmula para bebés prematuros o de bajo peso al nacer}

\author{
Antonio Lucas Ferreira Feitosa* \\ Gabriel Trevizani Depolli** \\ Jorge Nicolás Silva Flores*** \\ Monica Lopes de Assunção**** \\ Maria da Conceição Carneiro Pessoa de Santana*
}

\begin{abstract}
Basuki F, Hadiati DR, Turner T, McDonald S, Hakimi M. Dilute versus full-strength formula in exclusively formula-fed preterm or low birth weight infants. Cochrane Database of Systematic Reviews 2019, Issue 6. DOI: 10.1002/14651858.CD007263. pub3.
\end{abstract}

Bebês prematuros podem necessitar de ajustes para se alimentar nas primeiras semanas de vida, e isto se deve à incapacidade de coordenar respiração/sucção e deglutição antes de 34 semanas de idade gestacional ${ }^{1}$, fazendo com que o leite materno - o alimento personalizado para esse período, não possa vir a ser ofertado diretamente da mama, sendo necessária a utilização de sondas de alimen- tação orogástrica ou nasoentéricas a depender da prematuridade e/ou complicações associadas.

$\mathrm{Na}$ impossibilidade da oferta do leite humano, seja da própria mãe ou de doadora, o qual deve ser pasteurizado em Banco de Leite Humano, torna-se necessária a oferta de fórmula infantil modificada para prematuro. Contudo, sabe-se do potencial alergênico da proteína do leite de vaca contida nes-

\footnotetext{
* Universidade Estadual de Ciências da Saúde de Alagoas, AL, Brasil

** Universidade Federal do Espírito Santo, ES, Brasil

*** Fundación Regional de Parkinson del Maule

**** Universidade Federal de Alagoas, AL, Brasil

E-mail para correspondência: Antonio Lucas Ferreira Feitosa e-mail: tonyferr@outlook.com Recebido: 02/10/2019

Aprovado: $17 / 12 / 2019$
} 
sas formulações associado a um intestino neonatal imaturo e altamente susceptível a apresentar manifestações gastrointestinais, entre elas a enterocolite necrosante $(\mathrm{ECN})^{2}$. A ECN, geralmente, acomete prematuros com peso inferior a um quilograma e quinhentos gramas $(1,5 \mathrm{Kg})$. Acredita-se que um dos fatores de proteção contra o desenvolvimento da ECN é a oferta prolongada de leite materno pelo seu efeito protetor e estimulador de uma microbiota bifidogênica, parâmetro que não é visto em prematuros alimentados com fórmula, onde a incidência de enterocolite é maior.

Os autores do estudo "Dilute versus full-strength formula in exclusively formula-fed preterm or low birth weight infants" desenvolvido nas cidades de Yogy, Indonédia e em Melbourne, Austrália, ora resenhado, representam mais um exemplo do esforço atual da comunidade científica em determinar evidências de qualidade nos aspectos centrais da saúde e nutrição neonatal. A revisão sistemática foi desenvolvida seguindo os parâmetros da Cochrane Database of Systematic Reviews para encontrar e revisar estudos de alta qualidade tendo como objetivo: avaliar os efeitos da fórmula diluída versus fórmula total sobre a incidência de enterocolite necrosante, intolerância alimentar, ganho de peso, tempo de permanência no hospital e tempo para atingir a ingestão total de calorias em recém-nascidos pré-termo ou com baixo peso ao nascer. O objetivo secundário foi avaliar os efeitos de diferentes estratégias de diluição.

Os pesquisadores utilizaram os bancos de dados das Cochrane, Medline, Embase e Cumulative Index to Nursing and Allied Health Literature (CINAHL). A primeira base de dados foi analisada por busca padrão da "Cochrane Neonatal" através de seu cadastro central de ensaios clínicos controlados buscado no período de 1966 a 01 de outubro de 2018. Os dados pesquisados da Embase foram datados entre 1980 a $1^{\circ}$ de outubro de 2018 e os do banco CINAHL foram datados de $1982 \mathrm{a} 1^{\circ} \mathrm{de}$ outubro de 2018. Além disso, os ensaios clínicos em andamento e aqueles recentemente finalizados foram pesquisados e incluídos através de outras plataformas, como os registros da Organização Mundial de Saúde (OMS). A seleção incluiu estudos randomizados e quase randomizados (incluindo cluster-randomizado) que comparavam as dosagens de fórmula infantil em recém-nascidos prematuros e/ou com baixo peso ao nascer. Foram excluídos os bebês que receberam leite artificial como complemento ao leite materno. A escala GRADE foi usada para avaliar o risco de viés além de uma metodologia com modelo de meta-análise de efeitos fixos.

A partir disso, três estudos envolvendo 102 prematuros e/ou com baixo peso ao nascer foram incluídos ${ }^{3,4,5}$. Os estudos compararam a fórmula diluída (dobro de volume e metade da fórmula) com a fórmula de força total. Todos os estudos foram classificados como tendo risco de viés por fatores dos estudos, como a provável ausência de cegamento dos participantes. Resultados sobre a enterocolite necrosante não foram relatados em nenhum dos estudos. Ao combinar dois dos estudos incluídos, com 88 bebês, em uma meta-análise, os autores sugeriram que a fórmula diluída com o dobro do volume, ou seja, metade da força, teria menos episódios de resíduos gástricos por dia, além de menos episódios de resíduos até alcançar 100 $\mathrm{kcal} / \mathrm{kg}$, menor quantidade de vômitos por dia e menos ocorrências de distensão abdominal maior que $2 \mathrm{~cm}$. Os dados ainda sugerem que bebês alimentados com fórmula diluída de volume duplo e metade da força podem ter atingido uma ingestão de energia adequada mais cedo quando comparados aos bebês do grupo de força total, embora não tenha evidências de que existe diferença entre os grupos para ganho de peso após uma semana do início da alimentação intragástrica. Dentre os estudos analisados, não houve relatos de tempo de internação hospitalar. A técnica utilizada para a obtenção desses resultados foi a comparação de amostras de concentração, densidade energética e composição da fórmula fornecida em ambos os casos dentro do mesmo estudo.

Em todas as pesquisas incluídas, houve também alimentação parenteral, mas este tópico não foi explicado como parte dos objetivos indicados no início da investigação, o que pode ter influenciado os resultados. Nenhum dos estudos relatou efeitos de mortalidade, enterocolite necrosante, sepse ou neurodesenvolvimento, o que sustenta a hipótese de que não há evidências suficientes para estabelecer a causalidade entre o início da enterocolite necrosante e o tipo de alimentação (completa ou diluída), respondendo ao principal objetivo desta revisão. Os autores concluem o estudo afirmando que mesmo que os trabalhos analisados sejam estudos pequenos e antigos, trazem evidências, porém baixa, de que os bebês prematuros e/ou com baixo peso ao nascer alimentados com fórmulas diluídas 
atingem a ingestão enérgica adequada em menos tempo. Entretanto, a confiança nesse resultado é baixa para os autores. Esta limitação é explicada através da incerteza sobre o risco de viés e poucos dados disponíveis, por isso preconizam que mais ensaios clínicos randomizados são necessários para confirmar as hipóteses.

Com o estudo em questão, é notória a escassez literária sobre o tema abordado. Poucos estudos apresentaram as diferentes formas de diluição e seus efeitos, como nenhum abordou os efeitos da enterocolite necrosante nos participantes e sua relação com o tempo de internação hospitalar. Por isso, os estudos analisados são limitantes, o que, consequentemente, leva a limitação dessa revisão sistemática e a ausência de evidências consideradas ao final de baixa convicção. Esta revisão é importante e de grande relevância para a literatura internacional e nacional, pois parece que poucos autores publicam sobre o tema. A limitação da revisão não se dá pelo desempenho dos autores, o que é admirável, mas pelos poucos estudos sobre a temática. Além disso, os autores buscaram solucionar este problema selecionando também estudos em andamento ou recentemente concluídos. Notavelmente, a Cochrane exibiu resultados bem definidos e esclarecidos sobre o baixo número de artigos analisados, fornecendo aos leitores uma imersão ao conhecimento de alimentação de neonatos e seus fatores adversos em uma revisão ampla e objetiva.
Assim, como os autores sugerem, estudos randomizados e quasi-randomizados são necessários para: orientar a maneira correta de alimentação em hospitais e maternidades, revisões com maior quantidade de estudos científicos sobre o tema e conhecimento da população sobre os meios de alimentação e o que pode ocorrer com o recém-nascido prematuro e/ou de baixo peso que necessitam da alimentação por meio da fórmula.

\section{Referências}

1. Mangalat N.Pediatric Intestinal Failure: A Review of the Scope of Disease and a Regional Model of a Multidisciplinary Care Team.Mo Med. 2019 Mar-Apr; 116(2): 129-133.

2. Feldens Letícia, Souza João C.K. de, Fraga José C. Existe associação entre a localização da doença e a idade gestacional ao nascimento de recém-nascidos operados por enterocolite necrosante. J. Pediatr. (Rio J.) [Internet]. 2018 June [cited 2019 Aug 28]; 94( 3 ): 320-324

3. Anderson DM, Heidebrecht A, Annibale DJ. Formula concentration in the initial feeding of premature infants. Pediatric Research 1995; 37(4): 301.

4. Currao WJ, Cox C, Shapiro DL. Diluted formula for beginning the feeding of premature infants. American Journal of Diseases in Childhood 1988; 142(7): 730-1. PUBMED: 3381775]

5. Sarna MS, Saili A, Pandey KK, Dutta AK, Mullick DN. Premature infant feeding: role of diluted formula. Indian Pediatrics 1990; 27(8): 829-33. PUBMED: 2279807] 